\title{
Tsafon
}

Revue d'études juives du Nord

$77 \mid 2019$

Contribution à l'histoire des traductions juives de la Bible hébraïque

\section{La traduction comme rédemption}

De Moses Mendelssohn à Buber-Rosenzweig

\section{Dominique Bourel}

\section{(2) OpenEdition}

\section{Journals}

Édition électronique

URL : https://journals.openedition.org/tsafon/1745

DOI : $10.4000 /$ tsafon. 1745

ISSN : 2609-6420

Éditeur

Association Jean-Marie Delmaire

Édition imprimée

Date de publication : 1 septembre 2019

Pagination : $55-64$

ISSN : $1149-6630$

Référence électronique

Dominique Bourel, «La traduction comme rédemption », Tsafon [En ligne], 77 | 2019, mis en ligne le 12 septembre 2019, consulté le 24 juin 2021. URL : http://journals.openedition.org/tsafon/1745 ; DOI :

https://doi.org/10.4000/tsafon. 1745 


\title{
La traduction comme rédemption
}

\section{De Moses Mendelssohn à Buber-Rosenzweig}

\author{
Dominique Bourel*
}

Parmi les multiples traductions de la Bible des temps modernes, deux versions allemandes s'imposent par leur qualité intrinsèque et leur valeur mythique : celle de Moses Mendelssohn au XVIII ${ }^{\mathrm{e}}$ siècle et celle de Martin Buber et Franz Rosenzweig au XX ${ }^{\mathrm{e}}$ siècle.

En réalité, comme nous allons le voir, les deux projets sont opposés bien que fondés sur le même constat : en Allemagne, comme dans toute l'Europe, la Bible et sa science étaient confisquées par des savants chrétiens. Il importait donc de reprendre possession d'un texte non seulement hébraïque mais encore juif. Sur ce plan, ces deux traductions connurent d'ailleurs le même succès et elles sont très souvent citées. Sont-elles toujours lues?

\section{La Bible de Moses Mendelssohn}

Mendelssohn (1728/29-1786) voulait travailler à l'émancipation des juifs en Europe et, dans son cas précis, en Allemagne. Aussi traduisit-il le Pentateuque en hochdeutsch (haut allemand), transcrit avec des caractères hébraïques afin que les juifs (qui parlaient essentiellement le yiddish et l'écrivaient en caractères hébraïques) puissent apprendre la langue du pays dans lequel ils voulaient s'intégrer. Un prospectus pour

\footnotetext{
* Directeur de recherche émérite au CNRS. Centre Roland Mousnier (Sorbonne).
} 
obtenir des souscriptions fut diffusé dès 1778: Alim li-teroufa [Feuillets en guise de remède] et la parution à Berlin des divers volumes s'échelonna entre 1780 et 1783.

Mendelssohn n'hésita pas à utiliser les traductions chrétiennes, surtout celle de Luther et de Calvin. Outre cette double novation, il s'entoura de quelques collaborateurs (les Biouristes) pour mener à bien un commentaire [Be'ur], en hébreu celui-là, dans lequel étaient explicités ses choix de traduction. La recherche récente a fait des progrès significatifs dans l'intelligence de cette entreprise ${ }^{1}$. Dans sa lettre en hébreu du 25 mai 1779 à Avigdor Levi, Mendelssohn revient sur les raisons de son entreprise :

J'ai traduit l'Écriture en allemand non pas pour me faire remarquer par mon travail et me faire un nom dans le pays, ou bien dans l'espoir d'un gain matériel, mais pour mes fils que Dieu m'a gardés. Mon fils aîné est mort - une épreuve venue de Dieu - et il me resta seulement mon fils Joseph, - que Dieu affermisse son cœur avec Sa Torah -. Je lui donnai donc la traduction allemande afin qu'il puisse, par elle, comprendre le sens simple (pschat) de l'Écriture jusqu'à ce que l'enfant grandisse et comprenne par lui-même. ${ }^{2}$

Mendelssohn n'est pas opposé au travail de la philologie historicocritique qui naît exactement pendant cette période en Allemagne. Mais il croit dans l'origine divine de l'Écriture. Pour lui il n'y a aucun doute :

La langue sainte dans laquelle ont été écrits les vingt quatre livres que nous possédons aujourd'hui est la langue dans laquelle Dieu a parlé au premier homme (Adam) à Caïn, à Noé, aux patriarches ; dans laquelle il a fait entendre les Dix Paroles sur le mont Sinaï; dans laquelle ont été écrites les Tables de la Loi et dans laquelle il a parlé avec Moïse et avec ses prophètes.

Voilà ce qu'il écrit dans Or li-netiva [lumière sur le sentier], 1'expression est tirée du Ps.119: 105, son introduction aux volumes de sa Bible : Sefer Netivot ha-shalom [livre des sentiers de la paix]. Malgré une

\footnotetext{
${ }^{1}$ Outre l'excellente étude de Jean Lederman, La philosophie des lumières dans l'exégèse de Moses Mendelssohn, Paris, Champion, 2013, ainsi que celle d'Éli Schonfeld, L'apologie de Mendelssohn, Lagrasse, Verdier, 2018, et une traduction des Écrits juifs par René Lévy, Lagrasse, Verdier, 2018, on possède depuis peu une excellente anthologie due à Edward Breuer, Moses Mendelssohn's Hebrew Writings, New Haven and London Yale UP, 2018. Voir aussi le récent Moses Mendelssohn. Enlightenment, Religion, Politics, Nationalism, eds. Michah Gottlieb et Charles H. Manekin, Bethesda, Maryland, UP of Maryland, 2015.

${ }^{2}$ Moses Mendelssohn, Gesammelte Schriften, Jubiläumsausgabe, Bd. 19, p. 251-253 (en allemand, Bd. 20,2, p. 370-374).
} 
tradition déjà longue, il refuse d'employer dans sa traduction et son commentaire le judéo-allemand (ou yiddish) en raison de son caractère hybride, parce qu'il souhaite que les juifs utilisent soit l'allemand soit l'hébreu, qu'il connaît d'ailleurs parfaitement. En réalité l'expérience montre qu'il fallait déjà bien connaître l'allemand pour comprendre ce texte. Le lecteur juif déstabilisé, parce que néophyte en haut allemand, pouvait néanmoins s'appuyer sur le commentaire en hébreu.

Cette traduction fut critiquée par de hautes autorités rabbiniques, Ézéchiel Landau de Prague, Raphaël Cohen de Hambourg et Pinhas Hourwitz de Francfort, mais on pense aujourd'hui excessif de dire qu'ils auraient prononcé à son égard un herem, une excommunication. Le célèbre Moses Schreiber, le Hatam Sofer, était pourtant formel, enjoignant de «ne pas toucher aux livres de Mendelssohn»; ce qui n'empêcha pas son fils de souscrire à l'entreprise ! Cette dernière connut un succès évident en termes d'écho, de réédition et de débats. Mendelssohn était excellent prosateur, critique littéraire réputé, philosophe très célèbre qui avait remporté devant Kant le premier prix de la classe de philosophie spéculative de l'académie de Berlin. Son Phédon avait été traduit en dix langues avant la fin du XVIII ${ }^{\mathrm{e}}$ siècle! De plus il offrait en même temps, avec sa Jérusalem (1783), une véritable charte du judaïsme moderne, une séparation nette entre intégration et assimilation, et surtout une plaidoirie en faveur de la séparation de l'Église, du Temple et de la Synagogue d'avec l'État.

Un seul exemple peut suffire pour montrer la créativité de la Bible de Mendelssohn : Luther pour traduire la fameuse nomination de Dieu par lui-même dans Exode 3:14, donne : Ich werde sein der ich sein werde [je deviendrai celui que je deviendrai] ce que Mendelssohn rend par: Ich bin das Wesen welches ewig ist [Je suis l'Essence qui est éternelle]. Ce choix de traduction, où s'entend «l'Éternel » de Calvin et des huguenots présents à Berlin, a été très commenté. Mendelssohn s'en explique largement dans son Be'ur. Le tétragramme possède trois sens : pérennité, existence nécessaire et providence. Or il n'y a pas de terme allemand qui rende simultanément ewig, notwendig et vorsehend. Plus tard Rosenzweig, qui a consacré peu de temps avant sa mort un article à la question ${ }^{3}$, pense que Mendelssohn a mal tranché sous l'influence de Maïmonide et de Calvin.

\footnotetext{
${ }^{3}$ Franz Rosenzweig, «L'Éternel. Mendelssohn et le nom de Dieu» dans Franz Rosenzweig, L'Écriture, le verbe et autres essais, trad. Jean-Luc Evard, Paris, PUF, 1998, p. 113-130.
} 
Mais cette Bible et ses commentaires restent aujourd'hui encore un superbe témoignage du débat entre le judaïsme européen et les Lumières. Elle a aussi le mérite de lancer un véritable genre littéraire, la traduction de sources juives en allemand dont la chaîne est bien connue, qui commence avec Leopold Zunz et s'achève avec la traduction biblique dite de la communauté de Berlin durant la République de Weimar en passant par celles de Samson Raphael Hirsch et de Ludwig Philippson. Bien entendu, on reconnaît encore aujourd'hui l'aspect iconique de la traduction de Mendelssohn, plutôt citée comme un geste savant et intellectuel ouvrant sur un monde nouveau: la «symbiose » judéoallemande. Cette Bible a sans doute eu plus d'effet qu'on ne le croit, même sur les juifs d'Europe restés très observants. À lire la liste des souscripteurs que nous possédons, on ne peut lui dénier une certaine influence. En effet, non seulement l'Allemagne est bien présente, tout comme la France, mais aussi l'Europe orientale ${ }^{4}$.

\section{La Bible de Buber et Rosenzweig}

Martin Buber (1878-1965) et Franz Rosenzweig (1886-1929) s'attelèrent à une traduction visant l'effet inverse de celui recherché par Mendelssohn : faire entendre dans l'allemand un peu de l'hébreu disparu dans la perception chrétienne de la Bible hébraïque, médiatisée en Allemagne par Martin Luther et Jean-Sébastien Bach ${ }^{5}$. À partir de 1925 jusqu'à la mort de Rosenzweig (1929), les premiers volumes, publiés sans commentaires, offrirent une nouvelle traduction qui constitua un événement très débattu, dont l'audience dépassa largement le seul public juif cultivé. Buber poursuivit et acheva seul l'entreprise à Jérusalem, en 1961, suscitant l'un des plus beaux textes de Scholem, que nous lirons plus bas.

Lorsqu'il traduit la Bible avec Franz Rosenzweig, Martin Buber a déjà une longue carrière derrière lui : il est connu comme faisant partie

\footnotetext{
${ }^{4}$ Une des meilleures synthèses reste encore le chapitre dévolu à la Bible de Mendelssohn dans le classique Joseph Meisl, Haskalah. Geschichte der Aufklärungsbewegung unter den Juden in Russland, Berlin, 1919, rééditée et corrigée par Andreas Kennecke, Berlin, 2009.

${ }^{5}$ Il existe une importante littérature dans plusieurs langues sur cette traduction. Voir Maurice-Ruben Hayoun, Martin Buber - une introduction, Paris, Pocket Agora, 2013 et Dominique Bourel, Martin Buber. Sentinelle de l'humanité, Paris, Albin Michel, 2015. On possède désormais (2001-2019) une superbe édition presque achevée des œuvres de Martin Buber sous la direction de Bernd Witte et de Paul Mendes Flohr en 20 volumes, Martin Buber, Werkausgaben Gütersloh, Gütersloher Verlagshaus.
} 
de la jeune garde du sionisme, dans sa version humaniste et culturelle, et en tant que spécialiste du Hassidisme qu'il a contribué à faire redécouvrir. Il est un véritable héraut du « rajeunissement » du judaïsme en Allemagne et en Europe, notamment grâce à ses Discours sur le Judaïsme. C'est aussi un philosophe écouté depuis son retentissant Je et $T u$ (1923). Il enseigne d'ailleurs à Francfort à l'université et dans le Lehrhaus fondé par Rosenzweig. On connaît assez bien la genèse de leur traduction de la Bible, non seulement par l'abondante documentation rassemblée dans les archives Martin Buber de la Bibliothèque nationale de Jérusalem, mais encore par leur correspondance ${ }^{6}$.

C'est un jeune éditeur catholique, Lambert Schneider, qui prit l'initiative de cette nouvelle traduction en allemand:

Je ne sais pas si vous êtes convaincu de la nécessité d'une édition pratique de la Bible, en tout cas, aujourd'hui, une telle édition manque pour ceux qui en sont démunis. L'édition que je prévois doit bien évidemment être réalisée dignement, mais ne doit pas être un article de luxe que l'on met dans la bibliothèque mais que l'on n'ose pas lire. Je cherche un homme auquel je puisse confier la rédaction et je sais que, si vous acceptiez le travail, il serait entre les meilleures mains. C'est une grande sollicitation que je vous fais, c'est aussi un grand risque financier pour un jeune éditeur, mais j'espère quand même éveiller votre intérêt. (Lettre du 6 mai 1925).

Buber qui avait déjà eu un tel projet avant la Première Guerre mondiale n'hésita pas longtemps ; mais il demanda d'abord l'accord de Franz Rosenzweig afin de travailler avec lui. Buber fit régulièrement le voyage de Francfort afin de discuter péricope par péricope de la traduction avec l'aide du jeune Nahum Glatzer, d'autant plus que la maladie de Rosenzweig allait en s'aggravant. Dès la parution du premier volume en 1925, le débat s'embrasa dans toute l'Allemagne, juive et non juive, allant de l'enthousiasme public aux critiques véhémentes! Buber et Rosenzweig publièrent des réponses aux critiques et revinrent sur leurs principes $^{7}$. Comme l'écrivait Raphael Buber dans une lettre à son père (fin 1925), on lisait de l'allemand mais on entendait de l'hébreu. «C'est

\footnotetext{
${ }^{6}$ Voir les trois volumes publiés Briefwechsel, sous la direction de Grete Schaeder, Heidelberg, Kösel et Lambert Schneider 1972-1975. Nous citons, quand cela est possible, notre traduction avec Florence Heymann, Lettres choisies de Martin Buber 1899-1965, Paris, CNRS Éditions, 2004.

${ }^{7}$ Franz Rosenzweig, L'Écriture, le verbe et autres essais, tr. Jean-Luc Evard, Paris, PUF, 1998 ; Martin Buber, L'Écriture et sa traduction, tr. Marc B. de Launay et al., Paris, 2003. Buber a publié aussi d'autres ouvrages sur la Bible (La foi des prophètes, Moïse etc.) dans lesquels il répond à ses critiques.
} 
une double caractéristique qui distingue l'Écriture, ce qu'on appelle l'Ancien Testament, des grands livres des religions universelles. La première est qu'ici l'événement et la parole se situent résolument dans le peuple, dans l'histoire, dans le monde ». La seconde caractéristique est que cette écriture est une Loi. Mais surtout Buber insista toute sa vie sur le caractère oral de la Bible, c'est un livre qu'il faut lire qu'il faut entendre. La révélation s'incarne dans une voix. La lecture doit être une profération. Buber et Rosenzweig, très au fait de la science allemande, conservaient néanmoins pas mal d'humour, désignant la strate $R$ (Redaktor) par Rabbenou, notre maître !

« La structure des sons de l'allemand ne reproduira jamais celle des sonorités de l'hébreu, mais elle peut, développée à partir d'une impulsion analogue, exerçant un effet semblable, lui répondre en allemand, la germaniser » selon Buber. Paronomase, colométrie, réhébraïsation des noms propres, importance des racines, Buber invente toute une série de moyens pour faire claquer un texte presque anesthésié par les versions chrétiennes de Luther et de Bach. Il insiste sur la fonction hébraïque de la répétition de mots clés : «La phrase biblique entend être saisie de manière biblique, c'est-à-dire dans l'atmosphère produite par le retour des mêmes termes fondamentaux $\|^{8}$. Il faut déconceptualiser les mots, conserver le sens des rapports des termes entre eux. Dans ses livres Buber donne beaucoup d'exemples et dialogue avec de grands exégètes comme Hermann Gunkel et des exégètes juifs comme Benno Jacob, sans négliger non plus les versions catholiques puisqu'il a noué des contacts avec l'École biblique des dominicains de Jérusalem !

Comme exemples de leur méthode, rappelons Genèse $1: 2$. Là où Luther disait "Die Erde war wüst und leer», Buber et Rosenzweig conservant l'assonance de Tohu va-Vohu disent «Die Erde aber war Irrsal und Wirrsal». Le passage le plus célèbre est bien entendu Ex. 3 : 14, ehye asher ehye non pas tant «je suis celui qui suis »[Ich werde sein der ich sein werde] mais « je serai là en tant que moi qui serai là » [Ich werde dasein, als der ich dasein werde]. Buber précisait que plus qu'une traduction il s'agissait d'une "Verdeutschung », d'une germanisation du texte sacré. Buber n'a jamais cessé de réviser sa version allemande de la Bible, jusqu'à peu de temps avant sa mort !

\footnotetext{
${ }^{8}$ Gershom Scholem, "L'achèvement de la traduction de la Bible par Martin Buber » dans Le messianisme juif. Essai sur la spiritualité du judaïsme, tr. Bernard Dupuy, Paris, Calmann-Levy 1974, p. 441-447.
} 
Voici, comme nous l'annoncions plus haut, une partie de l'allocution de Scholem lors de la petite cérémonie organisée à Jérusalem, à l'occasion de la parution du dernier volume de la traduction complète de la Bible Buber-Rosenzweig, en février 1961.

Je ne sais plus exactement dans quelles circonstances, en 1924 ou 1925, vous avez décidé avec Rosenzweig de vous lancer dans cette entreprise. J'étais alors déjà en Palestine. Mais je crois vraiment que ce fut, comme cela arrive quelquefois, l'effet d'une volonté providentielle. [...] Quel défi pour quelqu'un comme vous que le texte même de l'écriture sainte ! Il faut plus que la tension de l'artiste et plus que la précision du philologue, surtout quand on reçoit le texte, comme Rosenzweig et vous l'avez fait, avec la volonté d'y reconnaître un point de vue spirituel et quand on est touché par lui. Vous avez mis beaucoup de vous même dans cette œuvre, même en ne voulant rien faire d'autre qu'une traduction la plus fidèle possible. [...] Si je considère l'intention première qui fut la vôtre et celle de Rosenzweig en vous lançant dans cette entreprise, je serais tenté de dire que vous vouliez adresser un appel au lecteur : mets-toi à apprendre l'hébreu ! Car votre traduction ne vise à aucun titre à rendre la Bible en un allemand qui en fournirait la claire compréhension par-delà toutes les difficultés. Au contraire, vous avez mis un soin particulier à ne pas rendre la Bible plus simple qu'elle n'est. Ce qui dans la Bible est clair, dans votre traduction demeure clair, ce qui est difficile demeure difficile et ce qui est incompréhensible demeure incompréhensible. Vous ne jetez pas le lecteur dans les illusions et vous ne lui faites pas de concessions. Le lecteur est sans cesse renvoyé à sa propre réflexion. Votre vœu c'est qu'il en vienne à se demander: qu'est-ce qui cherche à s'exprimer ici? Vous ne faites grâce de rien et vous ne rendez pas le texte plus facile. Au contraire, vous avez le sens aigu des obstacles et des difficultés qui se cache sous le flot apparemment limpide de la prose ou de la poésie. Je dirais presque que vous avez voulu rendre le texte plus ardu, afin que les mots ne cessent de toucher le lecteur toujours plus directement. La méthode que vous avez cru devoir retenir est la plus grande littéralité possible, une littéralité qui parfois paraît aller jusqu'à la limite et au-delà. Convaincu que la langue hébraïque est d'une structure abrupte, vous avez cherché à rendre cette structure dans votre traduction. Pas de conjonctions, pas de transitions lorsque l'hébreu n'en comporte pas! Pas de facilité avec un texte sublime! Vous avez voulu qu'il soit gardé dans son abrupte dignité.

Puis Scholem commente l'importance que Buber attachait à « la parole parlée » qui « cherche par tous les moyens à obliger le lecteur à lire le texte tout haut». Il ajoute que cette traduction est aussi un véritable commentaire.

Chaque fois qu'il nous est arrivé, à la plupart d'entre nous, de rencontrer dans la Bible des passages difficiles, nous nous sommes demandés : qu'est-ce que Buber dirait ici ? À peu près comme nous disions entre nous : qu'est-ce que Rachi en 
dit? Cette incorporation du commentaire dans la lettre même de la traduction me paraît un des résultats les plus remarquables de votre œuvre.

Marquant la longue interruption entre les débuts et la fin de la publication et les éditions différentes entre 1925 et 1961, Scholem achève par un beau compliment :

Il est désormais possible de lire le texte de la Bible en toute sérénité. La précision n'a pas été sacrifiée, mais on discerne maintenant une sorte de discrétion, qui vous fait adopter un style plus naturel, plus équilibré, celui de la parole parlée. Cela révèle une maîtrise qui se passe maintenant de ces audaces et atteint son but avec une certaine discrétion. Les mots du texte biblique ne sont plus constamment sous-tendus par ce qu'ils doivent évoquer, comme on l'éprouvait souvent dans la première traduction. C'est merveille que cette traduction soit devenue à présent une œuvre de maturité d'une telle pénétration exégétique et d'une telle fidélité linguistique.

Enfin il remarque que le nom de Dieu n'apparaît jamais comme tel dans cette traduction mais est remplacé par des pronoms, Je, Tu, Il.

Ce n'est pas là la moindre des nombreuses et audacieuses innovations de votre traduction. Elle repose sur votre conviction que, dans un livre qui parle du règne de Dieu dans la création et dans l'histoire le Nom de Dieu qu'ont connu les anciens écrivains bibliques n'a pas besoin de nous être livré, si ce n'est indirectement. Vous avez ainsi trouvé le moyen de concilier la traditionnelle crainte Dieu qui interdit de prononcer le Nom sacré et l'exigence de la parole biblique qui est de pouvoir être lue et ainsi entendue.

Les deux derniers paragraphes sont bien connus et tragiques : en effet lorsque cette traduction fut entreprise, association d'un sioniste et d'un non sioniste, on pouvait la considérer comme le cadeau de l'invité, Gastgeschenk offert par les juifs à l'Allemagne.

[...] mais les événements ont pris une tournure différente. Peut-être allez-vous vous récriez si je pose la question qui me vient invinciblement à l'esprit, mais je m'en réjouirais : à qui s'adresse à présent cette traduction et sur qui aura-t-elle de l'influence? Si l'on envisage les choses avec le regard de l'historien, cette traduction ne peut plus être le Gastgeschenk des juifs d'Allemagne. Elle sera plutôt - je dis cela non sans désagrément - elle sera la pierre tombale, Grabmal d'une relation qui a été anéantie dans une catastrophe effroyable. ${ }^{9}$

\footnotetext{
${ }^{9}$ Gershom G. Scholem, Le messianisme juif. Essai sur la spiritualité du Judaïsme, tr. Bernard Dupuy, Paris, Calmann Lévy, 1974, p. 440-447.
} 
Depuis plus de cinquante ans cette traduction a une autre histoire, qu'il faudra aussi suivre un jour ${ }^{10}$. Elle existe en livre de poche et elle est souvent citée, comme l'écrit Scholem, pour connaître les choix exégétiques de Buber, comme l'est celle d'André Chouraqui de nos jours.

Ainsi, les traductions de Mendelssohn et de Buber-Rosenzweig peuvent être aussi considérées comme un dialogue engagé avec le monde chrétien dans lequel nos auteurs vécurent, ainsi qu'un débat avec la science (philologie, histoire etc.) de ce monde. Il est frappant de constater en retour l'intérêt évident des traducteurs chrétiens pour ces versions juives.

\footnotetext{
${ }^{10}$ L'anniversaire de la traduction a donné lieu à deux colloques, à Heidelberg et à Heppenheim. Voir Daniel Krochmalnik et Hans-Joachim Werner (Hg.) 50 Jahre Martin Buber Bibel, Münster, Litt, 2014 ; et Andreas Losch, Thomas Reichert et Johannes Wassmer (Hg.) 'Alles in der Schrift ist echte Gesprochenheit'. Martin Buber und die Verdeutschung der Schrift, Lich, AV Verlag, 2016. Il existe des disques et CD de la Bible lue par Martin Buber et la Güterlsoher Verlagshaus a publié une superbe édition Die Schrift, avec illustrations de Marc Chagall, Gütersloh, 2007.
} 
Dominique Bourel 